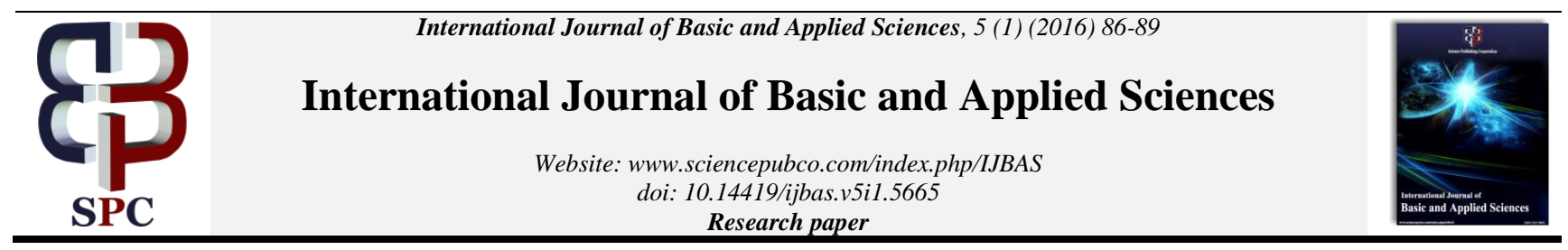

\title{
Exact solution of time-fractional partial differential equations using Laplace transform
}

\author{
Naser AlQutaifi * \\ Gulf University for Science \& Technology, Kuwait \\ *Corresponding author E-mail: alqautife@gmail.com
}

\begin{abstract}
The idea of replacing the first derivative in time by a fractional derivative of order $\alpha$, where $0<\alpha \leq 1$, leads to a fractional generalization of any partial differential equations of integer order. In this paper, we obtain a relationship between the solution of the integer order equation and the solution of its fractional extension by using the Laplace transform method.
\end{abstract}

Keywords: Abel's Integral Equation;Caputo's Fractional Derivative; Laplace Transform.

\section{Introduction}

As a generalization of the integer order partial differential equation, fractional partial differential equation is used to model important phenomena in various fields such as fluid flow, electromagnetic, acoustics, electrochemistry, cosmology, and material science. To find the explicit solutions of linear and nonlinear fractional partial differential equations, many powerful methods have been used such as the variational Iteration method, the homotopy perturbation method [10], the Laplace transform method [5], the Mellin transform method, the fractional complex transform method [3], [4] and the exponential function method [11].

In this paper, by Laplace transform method, we will obtain a relationship between the solution of the integer order partial differential equation and the solution of its fractional extension. In the following section we discuss the preliminaries. In Section 3, we apply the Laplace transform method to prove the main theorem of this paper, then we solved the time fractional Black-Scholes equation in Caputo's sense as an application for our results.

\section{Preliminaries}

\subsection{Caputo's fractional derivative}

Let $0<\alpha<1$ and $\mathrm{f}(\mathrm{t}) \in \mathrm{AC}[\mathrm{a}, \mathrm{b}]$, then the Caputo's fractional derivatives ${ }^{C} D_{a+}^{\alpha} f(t)$ and ${ }^{C} D_{b-}^{\alpha} f(t)$ are exist almost everywhere on $[a, b]$, and they are defined respectively by

${ }^{C} D_{a+}^{\alpha} f(t)=\frac{1}{\Gamma(1-\alpha)} \int_{a}^{t} \frac{f^{\prime}(\xi)}{(t-\xi)^{\alpha}} d \xi$

And

$$
{ }^{C} D_{b-}^{\alpha} f(t)=-\frac{1}{\Gamma(1-\alpha)} \int_{t}^{b} \frac{f^{\prime}(\xi)}{(\xi-t)^{\alpha}} d \xi .
$$

If $\alpha=n \in \mathbb{N}$ and the usual derivative $\mathrm{f}^{(\mathrm{n})}(\mathrm{t})$ of order $\mathrm{n}$ exists, then ${ }^{C} D_{a+}^{n} f(t)$ coincides with $f^{(n)}(t)$, while ${ }^{C} D_{b-}^{n} f(t)$ coincides with $\mathrm{f}^{(\mathrm{n})}(\mathrm{t})$ with exactness to the constant multiplier $(-1)^{\mathrm{n}}$. Thus, ${ }^{\mathrm{C}} \mathrm{D}_{\mathrm{a}+}^{\mathrm{n}} \mathrm{f}(\mathrm{t})=\mathrm{f}^{(\mathrm{n})}(\mathrm{t})$ and ${ }^{\mathrm{C}} \mathrm{D}_{\mathrm{b}-}^{\mathrm{n}} \mathrm{f}(\mathrm{t})=(-1)^{\mathrm{n}_{\mathrm{f}}}{ }^{(\mathrm{n})}(\mathrm{t})$. In particular, ${ }^{C} D_{a+}^{1} f(t)=f^{\prime}(t)$ and ${ }^{C} D_{b-}^{1} f(t)=-f^{\prime}(t)$. For more detailed information on the Caputo's fractional derivative, one can refer to [1], [8].

Now, we're going to introduce a lemma, but we'll use it later in section 3.

Lemma 2.1: ${ }^{C} D_{T-, t}^{\alpha} f(t)=a^{\alpha}{ }^{C} D_{0+, u}^{\alpha} g(u)$ where $f(t)=g(u)$ with $u=a(T-t)$ such that $0<t<T, 0<\alpha \leq 1$ and $a \in \mathbb{R}-$ $\{0\}$.

Proof:

Case (1): If $0<\alpha<1$.

Let $f(t)=g(u)$ with $u=a(T-t)$. Then by (2), we get

${ }^{\mathrm{C}} \mathrm{D}_{\mathrm{T}-, \mathrm{t}}^{\alpha} \mathrm{f}(\mathrm{t})=\frac{\mathrm{a}}{\Gamma(1-\alpha)} \int_{\mathrm{T}-\frac{\mathrm{u}}{\mathrm{a}}}^{\mathrm{T}} \frac{\mathrm{g}^{\prime}(\mathrm{a}(\mathrm{T}-\xi))}{\left(\frac{\mathrm{u}}{\mathrm{a}}-[\mathrm{T}-\xi]\right)^{\alpha}} \mathrm{d} \xi$

Now let $\mathrm{z}=\mathrm{a}(\mathrm{T}-\xi)$, thus

$$
{ }^{C} D_{T-, t}^{\alpha} f(t)=\frac{a^{\alpha}}{\Gamma(1-\alpha)} \int_{0}^{u} \frac{g^{\prime}(z)}{(u-z)^{\alpha}} d z
$$

Thus, from (1), we get ${ }^{\mathrm{C}} \mathrm{D}_{\mathrm{T}-\mathrm{t}}^{\alpha} \mathrm{f}(\mathrm{t})=\mathrm{a}^{\alpha}{ }^{\mathrm{C}} \mathrm{D}_{0+, \mathrm{u}}^{\alpha} \mathrm{g}(\mathrm{u})$ where $0<$ $\alpha<1$.

Case (2): If $\alpha=1$.

Then, $\mathrm{a}^{\mathrm{C}} \mathrm{D}_{0+, \mathrm{u}}^{1} \mathrm{~g}(\mathrm{u})$.

${ }^{\mathrm{C}} \mathrm{D}_{\mathrm{T}-, \mathrm{t}}^{1} \mathrm{f}(\mathrm{t})=-\mathrm{f}^{\prime}(\mathrm{t})=-\left[-\mathrm{ag}^{\prime}(\mathrm{u})\right]=\mathrm{ag}^{\prime}(\mathrm{u})=$ Therefore, ${ }^{\mathrm{C}} \mathrm{D}_{\mathrm{T}-\mathrm{t}}^{\alpha} \mathrm{f}(\mathrm{t})=\mathrm{a}^{\alpha}{ }^{\mathrm{C}} \mathrm{D}_{0+, \mathrm{u}}^{\alpha} \mathrm{g}(\mathrm{u})$ where $\mathrm{f}(\mathrm{t})=\mathrm{g}(\mathrm{u})$ with $\mathrm{u}=\mathrm{a}(\mathrm{T}-\mathrm{t})$ such that $0<\mathrm{t}<\mathrm{T}, 0<\alpha \leq 1$ and $\mathrm{a} \in \mathbb{R}-\{0\}$.

\subsection{Laplace transform}

The Laplace transform of a function $f(t)$ of a real variable $t \in \mathbb{R}^{+}$ is defined by

$\tilde{\mathrm{f}}(\mathrm{s})=\mathcal{L}\{\mathrm{f}(\mathrm{t}) ; \mathrm{s}\}=\int_{0}^{\infty} \mathrm{e}^{-\mathrm{st}} \mathrm{f}(\mathrm{t}) \mathrm{dt},(\mathrm{s} \in \mathbb{C})$. 
If the integral (5) is convergent at the point $s_{0} \in \mathbb{C}$, then it converges absolutely for $s \in \mathbb{C}$ such that $\operatorname{Re}(s)>\operatorname{Re}\left(s_{0}\right)$. The infimum $c_{f}$ of values $s$ for which the Laplace integral (5) converges is called the abscissa of convergence. Thus the Laplace integral (5) converges for $\operatorname{Re}(s)>c_{f}$ and diverges for $\operatorname{Re}(s)<c_{f}$. In other words, if the function $f(t)$ be continuous or piecewise continuous over every finite interval in $[0, \infty)$ and of exponential order $\mathrm{e}^{\text {at }}$, i.e., there exist constants $\mathrm{M}>0$ and $\mathrm{T}>0$ such that $|\mathrm{f}(\mathrm{t})| \leq \mathrm{Me}^{\text {at }}$ for all $\mathrm{t}>\mathrm{T}$, then the Laplace transform $\mathcal{L}\{\mathrm{f}(\mathrm{t}) ; \mathrm{s}\}$ of $\mathrm{f}(\mathrm{t})$ exists for all s provided $\operatorname{Re}(\mathrm{s})>\mathrm{a}$.

The original $f(t)$ can be restored from the Laplace transform $\tilde{f}(s)$ with the help of the inverse Laplace transform

$\mathrm{f}(\mathrm{t})=\mathcal{L}^{-1}\{\tilde{\mathrm{f}}(\mathrm{s}) ; \mathrm{t}\}=\frac{1}{2 \pi \mathrm{i}} \int_{c-\mathrm{i} \infty}^{\mathrm{c}+\mathrm{i} \infty} \mathrm{e}^{\mathrm{st}} \tilde{\mathrm{f}}(\mathrm{s}) \mathrm{ds}$,

Where $c=\operatorname{Re}(s)>c_{\mathrm{f}}$. The integral in (6) is also called Bromwich integral [1].

Now, we review some of the basic properties and results of the Laplace Transform. Suppose that $\mathcal{L}\{f(t) ; s\}=\tilde{f}(s)$ and $\mathcal{L}\{g(t) ; s\}=\tilde{g}(s)$, then the following statements hold [2]:

$\mathcal{L}\left\{t^{n} ; s\right\}=\frac{n !}{s^{n+1}}$, Where $(n=0,1,2,3, \ldots)$

$\mathcal{L}\left\{t^{a} ; s\right\}=\frac{\Gamma(a+1)}{s^{a+1}}$, Where $(a>-1)$

Laplace Transform of the $n-t h$ Derivative:

$\mathcal{L}\left\{f^{(n)}(t) ; s\right\}=$

$s^{n} \tilde{f}(s)-s^{n-1} f(0)-s^{n-2} f^{\prime}(0)$

$-\cdots \cdots-s f^{(n-2)}(0)-f^{(n-1)}(0)$,

Where $f^{(r)}(0)$ is the value of $f^{(r)}(t)$ at $t=0, r=0,1, \ldots,(n-$ 1) and $n \in \mathbb{N}$.

Convolution Theorem:

$\mathcal{L}\{f(t) * g(t) ; s\}=$

$\mathcal{L}\left\{\int_{0}^{t} f(t-\tau) g(\tau) d \tau ; s\right\}=\tilde{f}(s) \tilde{g}(s)$.

Laplace Transform of the Caputo's fractional derivative ${ }^{c} D_{0+}^{\alpha} f(t)$ :

$\mathcal{L}\left\{{ }^{C} D_{0+}^{\alpha} f(t) ; s\right\}=s^{\alpha} \tilde{f}(s)-s^{\alpha-1} f(0)$,

Where $0<\alpha \leq 1$

\subsection{Abel's integral equation}

The Abel's integral equation for any $\beta \in(0,1)$ is given by

$f(t)=\int_{0}^{t} \frac{\varphi(\tau)}{(t-\tau)^{\beta}} d \tau, x>0$,

Where the function $f(t)$ has a continuous derivative on some interval $[0, \beta][9]$.

However, the only solution of equation (12) is given by [6]

$\varphi(t)=\frac{1}{\Gamma(\beta) \Gamma(1-\beta)} \cdot \frac{d}{d t}\left(\int_{0}^{t} \frac{f(\tau)}{(t-\tau)^{1-\beta}} d \tau\right)$.

\section{Method of Laplace transform}

In this section, we introduce a new method for finding closedform solutions to boundary and initial value problems for time- fractional partial differential equations based on the Laplace transform method.

Consider the following initial value problem

$\frac{\partial u(x, t)}{\partial t}+L_{x} u(x, t)=0$

With

$u(x, 0)=f(x)$

Where $L_{x}$ is an arbitrary linear differential operator in $x$ (of any order in the derivatives) and $u(x, t)$ be a function of apace and time, such that $0<x<\infty$ and $0<t<T$.

Now, by taking the Laplace transform (5) for equation (14) with respect to $t$ and using (9), we get

$s \tilde{u}(x, s)=f(x)-L_{x} \tilde{u}(x, s)$,

Where $\mathcal{L}\{u(x, t) ; s\}=\tilde{u}(x, s)$.

Now, consider the following time-fractional initial value problem

${ }^{C} D_{0+, t}^{\alpha} u_{\alpha}(x, t)+L_{x} u_{\alpha}(x, t)=0$

With

$u_{\alpha}(x, 0)=f(x)$,

where $L_{x}$ is the same linear differential operator as before, ${ }^{C} D_{0+, t}^{\alpha}$ is the Caputo's fractional derivative of order $\alpha(0<\alpha<1)$, and $u_{\alpha}(x, t)$ be a function of apace and time, such that $0<x<\infty$ and $0<t<T$.

By applying the Laplace transform (5) for equation (17) with respect to $t$ and using (11), we get

$s^{\alpha} \widetilde{u_{\alpha}}(x, s)=s^{\alpha-1} f(x)-L_{x} \widetilde{u_{\alpha}}(x, s)$,

Where $\mathcal{L}\left\{u_{\alpha}(x, t) ; s\right\}=\widetilde{u_{\alpha}}(x, s)$.

Since the $L_{x}$ operator is the same in the two equations (14) and (17), therefore by the uniqueness of the Laplace transform, we conclude that

$L_{x} \tilde{u}(x, s)=L_{x} \widetilde{u_{\alpha}}(x, s)$.

Then, from (16), (19) and (20), we get

$\tilde{u}(x, s)=f(x)\left[s^{-1}-s^{\alpha-2}\right]+s^{\alpha-1} \widetilde{u_{\alpha}}(x, s)$.

Theorem 3.1: Suppose that $u(x, t)$ is the solution of the initial value problem (14) subject to the condition (15), and $u_{\alpha}(x, t)$ is the solution of the time-fractional equation (17) subject to the condition (18), then the solutions $u(x, t)$ and $u_{\alpha}(x, t)$ are related by

$u_{\alpha}(x, t)=\frac{1}{\Gamma(\alpha)} \cdot \frac{d}{d t}\left(\int_{0}^{t} \frac{u(x, \tau)}{(t-\tau)^{1-\alpha}} d \tau\right)+f(x)\left[1-\frac{t^{\alpha-1}}{\Gamma(\alpha)}\right]$

Where $0<\alpha \leq 1$.

Proof:

Case (1): If $0<\alpha<1$.

By using the Laplace inverse (6), (7), (8), and the convolution theorem (10), equation (21) becomes

$\int_{0}^{t} \frac{u_{\alpha}(x, \tau)}{(t-\tau)^{\alpha}} d \tau=\Gamma(1-\alpha)\left[u(x, t)-f(x)+\frac{t^{-\alpha+1}}{\Gamma(2-\alpha)} \cdot f(x)\right]$

And thus an Abel's integral equation for any $\alpha \in(0,1)$.Hence, from (13), we get 
$u_{\alpha}(x, t)=$

$\frac{\Gamma(1-\alpha)}{\Gamma(1-\alpha) \Gamma(\alpha)} \cdot \frac{d}{d t}\left(\int_{0}^{t} \frac{u(x, \tau)-f(x)+\frac{\tau^{-\alpha+1}}{\Gamma(2-\alpha)} \cdot f(x)}{(t-\tau)^{1-\alpha}} d \tau\right)$.

Thus,

$u_{\alpha}(x, t)=$

$\frac{1}{\Gamma(\alpha)} \cdot \frac{d}{d t}\left(\int_{0}^{t} \frac{u(x, \tau)}{(t-\tau)^{1-\alpha}} d \tau\right)-\frac{f(x)}{\Gamma(\alpha)} \cdot t^{\alpha-1}+$

$\frac{f(x)}{\Gamma(\alpha) \Gamma(2-\alpha)} \cdot \frac{d}{d t}\left(\int_{0}^{t}(t-\tau)^{\alpha-1} \tau^{-\alpha+1} d \tau\right)$.

Let $z=\frac{t-\tau}{t}$, then

$\int_{0}^{t}(t-\tau)^{\alpha-1} \tau^{-\alpha+1} d \tau=$

$t \int_{0}^{1} z^{\alpha-1}(1-z)^{(-\alpha+2)-1} d z=t \cdot B(\alpha, 2-\alpha)$,

Where $B(\cdot, \cdot)$ is the famous Beta function?

We know $B(x, y)=\frac{\Gamma(x) \Gamma(y)}{\Gamma(x+y)}$, therefore equation (25) becomes

$u_{\alpha}(x, t)=\frac{1}{\Gamma(\alpha)} \cdot \frac{d}{d t}\left(\int_{0}^{t} \frac{u(x, \tau)}{(t-\tau)^{1-\alpha}} d \tau\right)+f(x)\left[1-\frac{t^{\alpha-1}}{\Gamma(\alpha)}\right]$

Case (2): If $\alpha=1$.

We have ${ }^{C} D_{0+}^{1} u(x, t)=\frac{\partial u(x, t)}{\partial t}$, since $\alpha=1$. Therefore, equation (14) and equation (17) are the same subject to the same initial condition. Thus, must $u_{\alpha}(x, t)=u(x, t)$. Hence, the result valid for $\alpha=1$.

Therefore, equation (27) is true for any $\alpha \in(0,1]$.

Corollary 3.1: If $0<\alpha<1$, then $u_{\alpha}(x, t)={ }^{C} D_{0+, t}^{1-\alpha} u(x, t)+$ $u(x, 0)$.

Proof:

When $0<\alpha<1$, we have ${ }^{C} D_{a+}^{\alpha} y(t)=D_{a+}^{\alpha} y(t)-\frac{f(a)}{\Gamma(1-\alpha)}(t-$ $a)^{-\alpha}$, where $D_{a+}^{\alpha}$ is the Riemann-Liouville fractional derivative operator of order $\alpha(0<\alpha<1)$, and it is given by $D_{a+}^{\alpha} y(t)=$ $\frac{1}{\Gamma(1-\alpha)} \frac{d}{d t}\left(\int_{a}^{t} \frac{y(\xi)}{(t-\xi)^{\alpha}} d \xi\right)$.

Therefore, equation (27) becomes

$u_{\alpha}(x, t)=$

$D_{0+, t}^{1-\alpha} u(x, t)-\frac{u(x, 0)}{\Gamma(\alpha)} t^{-(1-\alpha)}+u(x, 0)$.

Thus,

$u_{\alpha}(x, t)={ }^{C} D_{0+, t}^{1-\alpha} u(x, t)+u(x, 0)$.

Now, consider the following boundary value problem

$\frac{\partial u(x, t)}{\partial t}-L_{x} u(x, t)=0$

With

$u(x, T)=g(x)$

Where $L_{x}$ is an arbitrary linear differential operator in $x$ (of any order in the derivatives) and $u(x, t)$ be a function of apace and time, such that $0<x<\infty$ and $0<t<T$.
Now, we need to reverse the direction of time, so that the terminal condition (31) becomes the initial condition for the given equation (30). Set $u(x, t)=v(x, z)$, where $z=T-t$; is a new time coordinate which runs over the interval $(0, T)$.

Thus, the boundary value problem becomes

$\frac{\partial v(x, z)}{\partial z}+L_{x} v(x, z)=0$

With

$v(x, 0)=g(x)$.

Now, consider the following time-fractional boundary value problem

$-{ }^{C} D_{T-, t}^{\alpha} u_{\alpha}(x, t)-L_{x} u_{\alpha}(x, t)=0$

With

$u_{\alpha}(x, T)=g(x)$

Where $L_{x}$ is the same linear differential operator as in (30), ${ }^{C} D_{T-, t}^{\alpha}$ is the Caputo's fractional derivative of order $(0<\alpha<1)$, and $u_{\alpha}(x, t)$ be a function of apace and time, such that $0<x<\infty$ and $0<t<T$.

We need to reverse the direction of time, so that the terminal condition (35) becomes the initial condition for the given equation (34). Set $u_{\alpha}(x, t)=v_{\alpha}(x, z)$, where $z=T-t$; is a new time coordinate which runs over the interval $(0, T)$. Hence, by lemma 2.1 , we have ${ }^{C} D_{T-, t}^{\alpha} u_{\alpha}(x, t)={ }^{C} D_{0+, z}^{\alpha} v_{\alpha}(x, z)$.

Thus, the given time-fractional boundary value problem becomes

${ }^{C} D_{0+, z}^{\alpha} v_{\alpha}(x, z)+L_{x} v_{\alpha}(x, z)=0$

With

$v_{\alpha}(x, 0)=g(x)$.

Theorem 3.2: Suppose that $u(x, t)$ is the solution of the boundary value problem (30) subject to the condition (31), and $u_{\alpha}(x, t)$ is the solution of the time-fractional equation (34) subject to the condition (35), then the solutions $u(x, t)$ and $u_{\alpha}(x, t)$ are related by

$u_{\alpha}(x, t)=-\frac{1}{\Gamma(\alpha)} \cdot \frac{d}{d t}\left(\int_{0}^{T-t} \frac{u(x, T-\tau)}{(T-t-\tau)^{1-\alpha}} d \tau\right)$

$+g(x)\left[1-\frac{(T-t)^{\alpha-1}}{\Gamma(\alpha)}\right]$

Where $0<\alpha \leq 1$.

Proof:

From theorem 3.1, we know that the solution of the initial value problem (32) subject to the condition (33), and the solution of the time-fractional equation (36) subject to the condition (37) are related by

$v_{\alpha}(x, z)=\frac{1}{\Gamma(\alpha)} \cdot \frac{d}{d z}\left(\int_{0}^{z} \frac{v(x, \tau)}{(z-\tau)^{1-\alpha}} d \tau\right)+g(x)\left[1-\frac{z^{\alpha-1}}{\Gamma(\alpha)}\right]$

Where $0<\alpha \leq 1$.

Since $u_{\alpha}(x, t)=v_{\alpha}(x, z)$, where $z=T-t$, then

$u_{\alpha}(x, t)=-\frac{1}{\Gamma(\alpha)} \cdot \frac{d}{d t}\left(\int_{0}^{T-t} \frac{u(x, T-\tau)}{(T-t-\tau)^{1-\alpha}} d \tau\right)$

$+g(x)\left[1-\frac{(T-t)^{\alpha-1}}{\Gamma(\alpha)}\right]$ 
Corollary 3.2: If $0<\alpha<1$, then $u_{\alpha}(x, t)={ }^{C} D_{T-, t}^{1-\alpha} u(x, t)+$ $u(x, T)$.

Proof:

When $0<\alpha<1$, we have ${ }^{C} D_{b-}^{\alpha} y(t)=D_{b-}^{\alpha} y(t)-\frac{f(b)}{\Gamma(1-\alpha)}(b-$ $t)^{-\alpha}$, where $D_{b-}^{\alpha}$ is the Riemann-Liouville fractional derivative operator of order $\alpha(0<\alpha<1)$, and it's given by $D_{b-}^{\alpha} y(t)=$ $-\frac{1}{\Gamma(1-\alpha)} \frac{d}{d t}\left(\int_{t}^{b} \frac{y(\xi)}{(\xi-t)^{\alpha}} d \xi\right)$.

Now, let $\xi=T-\tau$, then equation (38) becomes

$u_{\alpha}(x, t)=D_{T-, t}^{1-\alpha} u(x, t)-\frac{u(x, T)}{\Gamma(\alpha)}(T-t)^{\alpha-1}+u(x, T)$.

Hence,

$u_{\alpha}(x, t)={ }^{C} D_{T-, t}^{1-\alpha} u(x, t)+u(x, T)$.

The applicability of theorem 3.2 shall be demonstrated by the following example.

Example 3.1: Let $S$ denote the price of the stock, $K$ denote the strike of the option (exercise price), $r$ denote the risk-free interest rate, $\sigma$ denote the volatility of the stock's returns, and $t$ denote a time in years (assuming expiry $t=T$ ). In such setting, the time fractional Black-Scholes equation for the price of European put option $p_{\alpha}(S, t)$ reads as

$-{ }^{C} D_{T-, t}^{\alpha} p_{\alpha}(S, t)+\frac{\sigma^{2}}{2} S^{2} \frac{\partial^{2} p_{\alpha}(S, t)}{\partial S^{2}}$

$+r S \frac{\partial p_{\alpha}(S, t)}{\partial S}-r p_{\alpha}(S, t)=0$,

Where $0<t<T$ and $0<S<\infty$ subject to the boundary conditions

$\left\{\begin{array}{c}p_{\alpha}(S, T)=\theta(S)=(K-S)^{+}, \\ p_{\alpha}(0, t)=K e^{-r(T-t)},\end{array}\right.$

Where $\lim _{S \rightarrow \infty} p_{\alpha}(S, t)=0$ and ${ }^{C} D_{T-, t}^{\alpha}$ is the Caputo's fractional derivative with respect to $t ; 0<\alpha \leq 1$.

Solution:

Consider the following Black-Scholes equation for the price of European put option $p(S, t)$,

$\frac{\partial p(S, t)}{\partial t}+\frac{\sigma^{2}}{2} S^{2} \frac{\partial^{2} p(S, t)}{\partial S^{2}}+r S \frac{\partial p(S, t)}{\partial S}-r p(S, t)=0$,

Where $0<t<T$ and $0<S<\infty$ subject to the boundary conditions

$\left\{\begin{array}{c}p(S, T)=\theta(S)=(K-S)^{+} \\ p(0, t)=K e^{-r(T-t)}\end{array}\right.$

And $\lim _{S \rightarrow \infty} p(S, t)=0$.

From [7], we know that the exact solution of the above problem (45) is given by

$$
\begin{aligned}
& p(S, t)= \\
& \frac{e^{-\frac{1}{8}(T-t)\left(\sigma+\frac{2 r}{\sigma}\right)^{2} S\left(\frac{1}{2}-\frac{r}{\sigma^{2}}\right)}}{\sigma \sqrt{2 \pi(T-t)}} \cdot \int_{0}^{\infty} \theta(u) u^{-\left(\frac{3}{2}-\frac{r}{\sigma^{2}}\right)} e^{-\frac{\left(\ln \frac{S}{u}\right)^{2}}{2 \sigma^{2}(T-t)}} d u .
\end{aligned}
$$

Let $u=S e^{-(\sigma \sqrt{T-t}) \gamma}$, then

$p(S, t)=$
$\frac{e^{-\frac{1}{8}(T-t)\left(\sigma+\frac{2 r}{\sigma}\right)^{2}}}{\sqrt{2 \pi}} \cdot \int_{-\infty}^{\infty} \theta\left(S e^{-(\sigma \sqrt{T-t}) \gamma}\right) e^{\left(\frac{1}{2}-\frac{r}{\sigma^{2}}\right)(\sigma \sqrt{T-t}) \gamma} e^{-\frac{\gamma^{2}}{2}} d \gamma$

Now, let $z=-\gamma+\left(\frac{1}{2}-\frac{r}{\sigma^{2}}\right)(\sigma \sqrt{T-t})$. Thus

$p(S, t)=$

$\frac{e^{-r(T-t)}}{\sqrt{2 \pi}} \int_{-\infty}^{\infty} \theta\left(S e^{z(\sigma \sqrt{T-t})+\left(r-\frac{\sigma^{2}}{2}\right)(T-t)}\right) e^{-\frac{z^{2}}{2}} d z$.

Therefore, by theorem 3.2, equation (43) has a unique solution of the following form

$p_{\alpha}(S, t)=-\frac{1}{\Gamma(\alpha)} \cdot \frac{d}{d t}\left(\int_{0}^{T-t} \frac{p(S, T-\tau)}{(T-t-\tau)^{1-\alpha}} d \tau\right)$

$+\theta(S)\left[1-\frac{(T-t)^{\alpha-1}}{\Gamma(\alpha)}\right]$

Where $p(S, t)=\frac{e^{-r(T-t)}}{\sqrt{2 \pi}} \int_{-\infty}^{\infty} \theta\left(S e^{z(\sigma \sqrt{T-t})+\left(r-\frac{\sigma^{2}}{2}\right)(T-t)}\right) e^{-\frac{z^{2}}{2}} d z$

\section{Conclusion}

If we know the solutions of the integer order partial differential equation, then by our results it is easy to obtain an exact and explicit analytic solutions of its fractional extension in Caputo's sense.

\section{References}

[1] Kilbas A., Srivastava H. \& Trujillo J., Theory and Applications of Fractional Differential Equations, Elsevier, USA, 2006.

[2] Debnath L. \& Bhatta D., Integral Transforms and Their Applications, Chapman \& Hall/ CRC, Boca Raton, 2006.

[3] Li Z. \& He J., Fractional complex transformation for fractional differential equations, Math. Comput. Appl., Vol.15, (2010), pp.970973.

[4] Li Z. \& He J., Converting Fractional differential equations into partial differential equations, Thermal Science, (2012), DOI REFERENCE: $10.2298 /$ TSCI1 10503068H.

[5] Kazem S., Exact Solution of Some Linear Fractional Differential Equations by Laplace Transform, International Journal of Nonlinear Science, Vol.16, No.1, (2013), pp.3-11.

[6] Krasnov M., Kiselev A. \& Makarenko G., Problems and Exercises in Integral Equations, MIR Publishers, Moscow, 1971.

[7] Kalla S. \& Chawla M., Analytical and numerical approach to financial derivatives-An overview, Bull. Pure Appl. Math., Vol.4, No.1, (2010), pp.20-33.

[8] Miller K. \& Ross B., An Introduction to the Fractional Calculus and Fractional Differential Equations, John Wiley \& Sons Inc., USA, 1993.

[9] Samko S., Kilbas A. \& Marichev O., Fractional Integrals and Derivatives: Theory and Applications, Gordon and Breach Science Publishers, Amsterdam, 1993.

[10] Liao S., on the homotopy analysis method for nonlinear problems, Appl. Math. Comput, Vol.147, (2004), pp.499-513. http://dx.doi.org/10.1016/S0096-3003(02)00790-7.

[11] Wu X. \& He J., Exp-function method and its application to nonlinear equations, Chaos, Solitons and Fractals, Vol.38, No.3, (2008), pp.903-910. http://dx.doi.org/10.1016/j.chaos.2007.01.024. 